\title{
Prenatal food restriction induces poor-quality articular cartilage in female rat offspring fed a post-weaning high-fat diet and its intra-uterine programming mechanisms
}

\author{
Yang Tan ${ }^{1,2}$, Yunpeng $\mathrm{Wu}^{1}$, Qubo $\mathrm{Ni}^{1}$, Yu Deng ${ }^{1}$, Jing $\mathrm{Li}^{3}$, Linlong Wang ${ }^{1}$, Lang Shen ${ }^{3}$, Yansong Liu ${ }^{3}$, \\ Jacques Magdalou ${ }^{4}$, Hui Wang ${ }^{3,2}$ and Liaobin Chen ${ }^{1,2 *}$ \\ ${ }^{1}$ Department of Orthopedic Surgery, Zhongnan Hospital of Wuban University, Wuban 430071, People's Republic of China \\ ${ }^{2} H u b e i$ Provincial Key Laboratory of Developmentally Originated Disease, Wuban 430071, People's Republic of China \\ ${ }^{3}$ Department of Pharmacology, Basic Medical School of Wuban University, Wuban 430071, People's Republic of China \\ ${ }^{4}$ Université de Lorraine, Ingénierie Moléculaire, Physiopathologie Articulaire (IMoPA), UMR 7365 CNRS, Biopôle, F-54505 \\ Vandoeuvre-lès-Nancy, France
}

(Submitted 7 January 2016 - Final revision received 10 August 2016 - Accepted 22 August 2016 - First published online 29 September 2016)

\section{Abstract}

Epidemiological data show that osteoarthritis (OA) is significantly associated with lower birth weight, and that OA may be a type of fetaloriginated adult disease. The present study aimed to investigate the prenatal food-restriction (PFR) effect on the quality of articular cartilage in female offspring to explore the underlying mechanisms of fetal-originated OA. Maternal rats were fed a restricted diet from gestational day (GD) 11 to 20 to induce intra-uterine growth retardation. Female fetuses and female adult offspring fed a post-weaning high-fat diet were killed at GD20 and postnatal week 24, respectively. Serum and knee cartilage samples from fetuses and adult female offspring were collected and examined for cholesterol metabolism and histology. Fetal serum corticosterone and insulin-like growth factor-1 (IGF-1) in the PFR group were lower than those of the control, but the serum cholesterol level was not changed. The lower expression of IGF-1 in the PFR group lasted into adulthood. The expression of extracellular matrix (ECM) genes, including type II collagen, aggrecan and cholesterol efflux genes including liver X receptor, were significantly induced, but the ATP-binding-cassette transporter A1 was unchanged. PFR could induce a reduction in ECM synthesis and impaired cholesterol efflux in female offspring, and eventually led to poor quality of articular cartilage and OA.

Key words: Prenatal food restriction: Articular cartilage: Glucocorticoids: Insulin-like growth factor-1: Cholesterol

Dysplasia of fetal cartilage will affect the quality of cartilage after birth and result in a predisposition to osteoarthritis (OA) or premature cartilage degeneration ${ }^{(1,2)}$. Therefore, more and more attention has been drawn to the correlations between OA and factors of nutrition and environment in the critical early development period.

Epidemiological data show that OA is significantly associated with lower birth weight ${ }^{(3,4)}$, and that OA may be a type of fetaloriginated adult disease ${ }^{(5)}$. Other investigations have indicated that offspring with intra-uterine growth retardation (IUGR) have an enhanced susceptibility to the metabolic syndrome (MS), including dyslipidaemia and coronary arterial atherosclerosis $^{(6,7)}$. IUGR is defined as a birth weight and/or length below the 10th percentile for gestational age and an abdominal circumference below the $2 \cdot 5$ th percentile with pathological restriction of fetal growth ${ }^{(8)}$. The correlation between the MS and OA has been highlighted in the aetiological study of OA, and many researchers' opinions about $\mathrm{OA}$ is that it is characterised as a metabolic disorder ${ }^{(9)}$. OA has a close relation to CVD and serum cholesterol levels ${ }^{(10-14)}$. Therefore, IUGR in offspring increases the risk of adult MS; specifically, hyperlipidaemia may affect the quality of cartilage. The intra-uterine programmed alteration is defined as an environmental factor operating early in life that potently affects the developing system, permanently altering its structure and function for life ${ }^{(15-18)}$. It is widely accepted that IUGR's original programmed metabolic dysfunctions and susceptibility to MS are induced by overexposure to maternal glucocorticoids (GC) in the fetus ${ }^{(19)}$. Our previous study found that adverse exogenous environments during pregnancy may lead to fetal overexposure to exogenous and maternal GC by affecting the placental barrier and sequentially suppressing the fetal hypothalamic-pituitary-adrenal (HPA) axis and may result in

Abbreviations: ABCA1, ATP-binding-cassette transporter A1; Col2a1, collagen type II $\alpha 1$; GC, glucocorticoids; GD, gestational day; HFD, high-fat diet; IGF-1, insulin-like growth factor-1; IUGR, intra-uterine growth retardation; LXR, liver X receptor; MS, metabolic syndrome; OA, osteoarthritis; PFR, prenatal foodrestriction. 
growth retardation ${ }^{(20-23)}$. We also confirmed that prenatal food-restriction (PFR) can induce lower birth weight and altered glucose/lipid metabolism by the programmed alteration of the HPA axis-associated neuroendocrine metabolism ${ }^{(24)}$. Therefore, IUGR in offspring increases the risk of adult hyperlipidaemia and may be responsible for the IUGR-induced poor quality of articular cartilage.

Insulin-like growth factor-1 (IGF-1) is an important factor for the development of the fetal skeletal muscle system, especially in cartilage growth regulation ${ }^{(25)}$. IGF-1 can promote individual growth and development by regulating cell proliferation and apoptosis through the phosphorylation of mitogen-activated protein kinase (MEK) and extracellular regulated kinase (ERK); it can also promote the synthesis of cartilage matrix collagen type II $\alpha 1$ (Col2a1) and proteoglycan through phosphorylation of phosphatidylinositol 3-kinase/protein kinase B (PI3K/Akt) ${ }^{(26,27)}$. During fetal development, IGF-1 in articular cartilage is mainly obtained from the fetal liver ${ }^{(28)}$. Cartilage can gradually build paracrine and autocrine functions of IGF-1 in the late development stage $^{(29)}$. IGF-1 is regulated by various cytokines and hormones; GC plays an important role in the regulation of synthesis and secretion of IGF-1 during the embryonic period. High levels of GC could inhibit bone and cartilage physiological function by inhibiting IGF-1 secretion ${ }^{(30,31)}$. Therefore, we propose that GC may induce cartilage dysplasia and its low function in IUGR by inhibiting fetal chondrocyte proliferation and by down-regulating the IGF-1 signalling pathway.

Intracellular cholesterol in peripheral tissues is mainly converted by ATP-binding-cassette transporter A1 (ABCA1) to ApoA1, a form of HDL-cholesterol released into the blood and transferred to the liver. This process is mediated by transcriptional factor PPAR and liver $\mathrm{X}$ receptor $(\mathrm{LXR})^{(32)}$. Similar to atherosclerosis, inhibition of the cholesterol efflux system in OA patients' chondrocytes, characterised as lower LXR expression, leads to cellular cholesterol accumulation in cells ${ }^{(9)}$. Therefore, hypercholesteraemia and the cholesterol efflux dysfunction in offspring with IUGR may be responsible for the poor quality of articular cartilage in adults.

Under conditions of postnatal over-nutrition, offspring with IUGR usually exhibited rapid catch-up growth, directly shown as children's rapid growth and development, such as a body weight growth rate significantly higher than that of normal children, which increased susceptibility to aggravated glucose and lipid metabolic disorders, leading to an increased susceptibility to adult $\mathrm{MS}^{(33)}$. An adverse intra-uterine environment will result in increased sensitivity of the peripheral tissues to metabolic hormones (e.g. GC) in offspring; this latter condition enhances survival and maximises growth and fuel deposition as the nutritional pattern improves after birth. If postnatal nutrient availability is greater than that predicted prenatally, enhanced postnatal growth and fat deposition will occur ${ }^{(34)}$. In order to simulate a nutrient-rich environment, the offspring with IUGR were fed a post-weaning high-fat diet (HFD) to magnify PFR-induced adult dyslipidaemia and help investigate the correlation between IUGR and chondrogenesis retardation.

The prevalence of OA was substantially higher in women than in men ${ }^{(35,36)}$. Moreover, other studies and our results indicated that female rat offspring with IUGR were more susceptible to diet-induced dyslipidaemia ${ }^{(37-39)}$. Therefore, we chose female rats as the research subjects in this study. In the present study, we used a PFR-induced IUGR model in female rats to explore the effect of a poor environment in utero on cartilage development, and tried to clarify the correlation between dyslipidaemia and poor cartilage quality in offspring with IUGR using a post-weaning HFD.

\section{Methods}

\section{Materials}

Isoflurane was purchased from Baxter Healthcare Co. Rat corticosterone (CORT) ELISA kits were obtained from Assaypro LLC. Rat IGF-1 ELISA kits were purchased from RD Systems Inc. Total cholesterol (TCH), LDL-cholesterol and HDL-cholesterol assay kits were purchased from Sangon Biotech Co. Ltd. Reverse transcription kits were purchased from TaKaRa Biotechnology Co. Ltd. GeXP multiplex gene expression analysis kits were purchased from Beckman Coulter Inc. The oligonucleotide primers for rat GeXP multiplex gene expression analysis (HPLC purification) were synthesised by Sangon Biotech Co. Ltd. Polyclonal antibodies for Col2a1, IGF-1, LXR and ABCA1 were obtained from Santa Cruz Biotechnology Inc. Other chemicals and agents used were of analytical grade.

\section{Animals and treatment}

The animal experiments were performed at the Center for Animal Experiment of Wuhan University (Wuhan, China), which has been accredited by the Association for Assessment and Accreditation of Laboratory Animal Care International. All animal experimental procedures were approved by the Chinese Animal Welfare Committee and performed in accordance with the Guidelines for the Care and Use of Laboratory Animals of the Chinese Animal Welfare Committee.

Specific pathogen-free Wistar rats (females weighing 200-240 g and males weighing 260-300 g) were obtained from the Experimental Center of Hubei Medical Scientific Academy (no. 2009-0004, Hubei, China). Animals were housed (room temperature: $18-22^{\circ} \mathrm{C}$; humidity: $40-60 \%$ ), acclimated and mated. Upon confirmation of mating by the appearance of sperms in a vaginal smear, the day was considered as gestational day (GD) 0. Pregnant females were then transferred into individual cages. Pregnant rats were randomly divided into a control group and a food-restriction group. At study start, the food-restriction group was fed a restricted diet (50\% of the daily food intake of the control rats) from GD11 until term delivery $(G D 20)^{(19)}$. The control group was allowed normal food ad libitum. On GD20, eight randomly selected pregnant rats from each group were anaesthetised with isoflurane and euthanised. Female fetuses were quickly removed and weighed; the litter size of each pregnant rat was set to ten to fourteen live fetuses. Female fetal blood and serum samples were collected. Female fetal bilateral tibial knee cartilage and right femoral knee cartilage were separated under a dissection microscope and collected. The samples collected from each littermate were pooled together and immediately frozen in 
liquid $\mathrm{N}_{2}$, followed by storage at $-80^{\circ} \mathrm{C}$ for subsequent analyses. Fetal left femoral knee cartilage was randomly selected (one per litter) and fixed in phosphate-buffered $4 \%$ paraformaldehyde solution for $24 \mathrm{~h}$ before being decalcified in EDTA, dehydrated in alcohol and embedded in paraffin.

The other pregnant rats (eight for each group) were maintained until normal delivery on GD21. On postnatal day 1, female pups were normalised into eight pups per litter to assure adequate and standardised nutrition until weaning (postnatal week 4, PW4). After weaning, the female pups were randomly selected and assigned into two groups: a control group and a PFR group. All pups were weaned to an ad libitum HFD before being killed on PW24. The HFD was previously reported by our laboratory $^{(24)}$ and provided $18.9 \%$ of its energy content as protein, $61.7 \%$ as carbohydrate and $19.4 \%$ as fat. The body weights of the rat offspring were measured weekly. At PW24, the offspring were anaesthetised with isoflurane and decapitated in a room separate from that in which the other animals were maintained. Serum was prepared and stored at $-80^{\circ} \mathrm{C}$ until further analysis. The left femoral knee cartilage was dissected and fixed in $4 \%$ paraformaldehyde solution for $24 \mathrm{~h}$ and decalcified in $10 \%$ EDTA for $21 \mathrm{~d}$ for histological examination.

\section{Histological measurement and immunohistochemical assays}

For histological analysis, sections of fetal and adult femoral cartilage were stained with safranin $O$. Femoral knee cartilage sections of adult offspring were also stained with toluidine blue (TB). The sections were observed and photographed using a Nikon ECLIPSE-Ci light microscope (Nikon). Cartilage damage was scored according to modified Mankin's score as reported by Henson ${ }^{(40,41)}$. Cartilage cell number, matrix structure, matrix staining and tidemark were graded on the basis of four parts of the cartilage: the superficial layer, the transitional zone, the radial zone and the tidemark zone of calcified cartilage.

Immunohistochemical assays were performed to determine the expression levels of IGF-1, Col2a1, LXR and ABCA1 proteins. Femoral knee cartilage was fixed in $10 \%$ formaldehyde for $24 \mathrm{~h}$, after which staining for IGF-1, Col2a1, LXR and ABCA1 was performed using the immunohistochemical streptavidin-biotin-peroxidase complex method with polyclonal antibodies against IGF-1, Col2a1, LXR and ABCA1 (diluted 1:200), with normal saline as the negative control. Positive expression was determined by the presence of brown granules. All images were captured using a Nikon ECLIPSE-Ci Light Microscope. Analysis of the stained images was conducted using NIS-Elements BR, Nikon (version 4.2). The intensity of staining was determined by measuring the integral optical density (IOD) in ten different fields for each section. The average IOD for each sample was calculated from three sections. In adult sections, the region of interest (ROI) was the stained cartilage. In fetal sections, the ROI was the stained epiphysis (the rounded end of the long bone) of the femur.

\section{Multiplex gene expression analysis}

The gene expression profile of fetal femoral knee cartilage was detected by multiplex gene expression analysis, which was performed in our previous study ${ }^{(42)}$. The list of genes and primer pairs are given in Table 1. Relative RNA expression levels were normalised to glyceraldehyde 3-phosphate dehydrogenase, $\beta$-actin and tubulin expression levels.

\section{Analysis for blood samples}

Serum CORT and IGF-1 concentrations were detected using ELISA kits. TCH, LDL-cholesterol and HDL-cholesterol concentrations were detected using biochemical assay kits.

\section{Statistical analysis}

SPSS 17 (SPSS Science Inc.) was used for data analysis. Quantitative data were expressed as means with their standard errors and were evaluated using Student's two-tailed

Table 1. Genes and primer pairs for the GeXP Genetic Analysis System

\begin{tabular}{|c|c|c|}
\hline Genes & Forward primers & Reverse primers \\
\hline IGF-1 & AGGTGACACTATAGAATACCAGCTGTTTCCTGTCTACAG & GTACGACTCACTATAGGGAAGATGTGAAGACGACATGATGT \\
\hline IGF-1R & AGGTGACACTATAGAATACAAGACAGAAGTCTGCGGTG & GTACGACTCACTATAGGGACCGGGTCTGTGATATTGTAGG \\
\hline MEK1 & AGGTGACACTATAGAATACAAGATGCCCAAGAAGAAGC & GTACGACTCACTATAGGGAAGCCATAACCAGGCCAGAT \\
\hline MEK2 & AGGTGACACTATAGAATATCATTAAGAACCCAGCAGAGC & GTACGACTCACTATAGGGATCTCCACTTTGTTTGCTTTTCC \\
\hline PI3K & AGGTGACACTATAGAATACGATGGAATTGGAACGAGTG & GTACGACTCACTATAGGGACCAGACTTTCAAGTCGTGCA \\
\hline AKT & AGGTGACACTATAGAATAATGAACGACGTAGCCATTGTG & GTACGACTCACTATAGGGAATGATGAAGGTGTTGGGCCT \\
\hline Aggrecan & AGGTGACACTATAGAATACGGACTGAAGTTCTTGGAGG & GTACGACTCACTATAGGGAGGTTGAGGGATGCTCACACT \\
\hline Col2a1 & AGGTGACACTATAGAATATCAAGGAGAAGCTGGACAGAA & GTACGACTCACTATAGGGACCCAGGGTTGCCATTAGAAC \\
\hline LDLR & AGGTGACACTATAGAATAACATTTCGGGTCTGTGATCC & GTACGACTCACTATAGGGACTGGAACTCGTTCTTGCCAC \\
\hline PPAR $y$ & AGGTGACACTATAGAATAGGCGATCTTGACAGGAAAGA & GTACGACTCACTATAGGGAGAAACTGGCACCCTTGAAAA \\
\hline LXR $\beta$ & AGGTGACACTATAGAATACCATTTCCAGGGTAACGAAG & GTACGACTCACTATAGGGAAGACATAGTGGGTCACGAAGC \\
\hline ABCA1 & AGGTGACACTATAGAATACGTCCTTGTGTCCATCTGTG & GTACGACTCACTATAGGGAAAGGGCTAGAACAGGCAGGT \\
\hline$\beta$-Actin & AGGTGACACTATAGAATAGTCCACCCGCGAGTACAAC & GTACGACTCACTATAGGGACCCACGTAGGAGTCCTTCTG \\
\hline GAPDH & AGGTGACACTATAGAATATCTCTGCTCCTCCCTGTTCTAG & GTACGACTCACTATAGGGAGGTCAATGAAGGGGTCGTTG \\
\hline Tubulin & AGGTGACACTATAGAATATGTAAGAAGCAACACCTCCTC & GTACGACTCACTATAGGGACAGTGCGAACTTCATCAATAAC \\
\hline
\end{tabular}

IGF-1, insulin-like growth factor-1; IGF-1R, insulin-like growth factor-1 receptor; MEK1, mitogen-activated protein kinase 1; MEK2, mitogen-activated protein kinase 2; PI3K, phosphatidylinositol 3-kinase; Akt, protein kinase B; Col2a1, collagen type II a1; LDLR, LDL receptor; LXR $\beta$, liver X receptor $\beta$; ABCA1, ATP-binding cassette transporter A1; GAPDH, glyceraldehyde 3-phosphate dehydrogenase. 
$t$ test. Mankin's Score was evaluated using the Mann-Whitney test. Statistical significance was defined as $P<0 \cdot 05$.

\section{Results}

\section{Body weights in fetuses and offspring}

At GD20, fetal weights in the PFR group were significantly lower compared with the control group $(P<0 \cdot 01$, Fig. 1(a)); body weights of female offspring in the PFR group were dramatically lower compared with the control group at the 1st, 4th, 8th and 12th weeks. However, in the 20th and 24th weeks, there were no significant differences between the two groups $(P<0 \cdot 01$, Fig. 1(a)). As seen in Fig. 1(b), the body weight increase ratio in the PFR group was higher compared with controls, especially at the 20th and 24th weeks $(P<0 \cdot 01)$. These results suggest that the IUGR and catch-up growth rat model induced by PFR was successfully established.

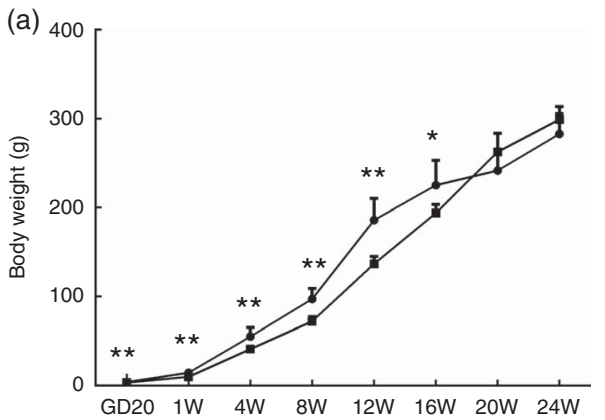

\section{Pathological change of articular cartilage in fetuses and adult offspring}

Examination of female fetal femoral knee cartilage (Fig. 2(a)) showed that the chondrocytes were scrambled in the PFR group. The fetal rats' articular cartilage matrix safranin $O$ staining was shallow; optical density analysis for safranin $O$ staining showed that PFR significantly decreased fetal rat articular cartilage matrix glycosaminoglycans levels (Fig. 2(b)).

After safranin $O$ staining examination of adult female offspring femoral knee cartilage, there were smooth surfaces and clear structures in the control group, and there were abundant chondrocytes with no clustering in the superficial layer or loss. Compared with the control group, the femoral cartilage matrix structure was disorganised with little cellular proliferation, and the tidemarks were disrupted in the PFR group (Fig. 2(c)). After TB staining, the articular cartilage had even dying and neat

(b)

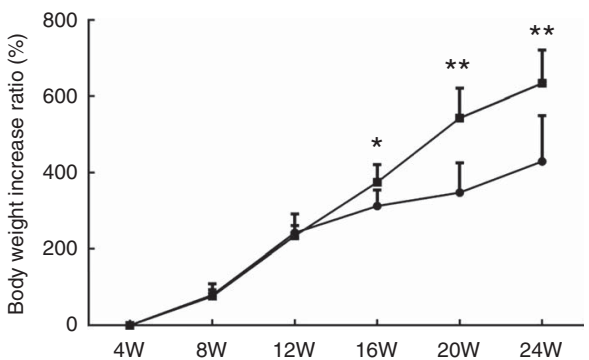

Fig. 1. Effects of prenatal food restriction - $\rightarrow$, and a post-weaning high-fat diet on offspring body weights. (a) Body weight from gestational day 20 (GD20) to postnatal week 24 (24W). (b) Body weight increase ratio, after weaning from 4 weeks to 24 weeks. - - Control group. Values are means $(n 8)$, with standard errors represented by vertical bars. Mean value was significantly different from that of the control group: ${ }^{\star} P<0.05$, ${ }^{* \star} P<0.01$.

(a)

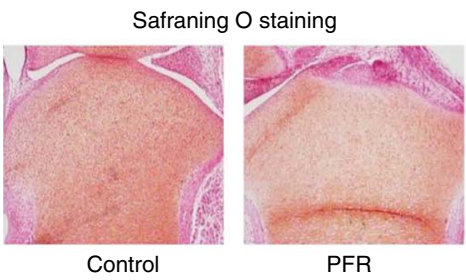

(b)

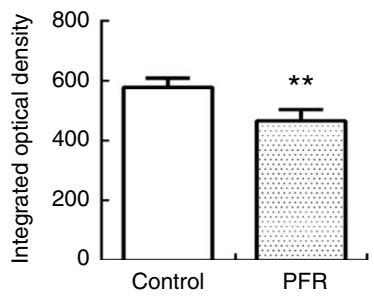

(c)
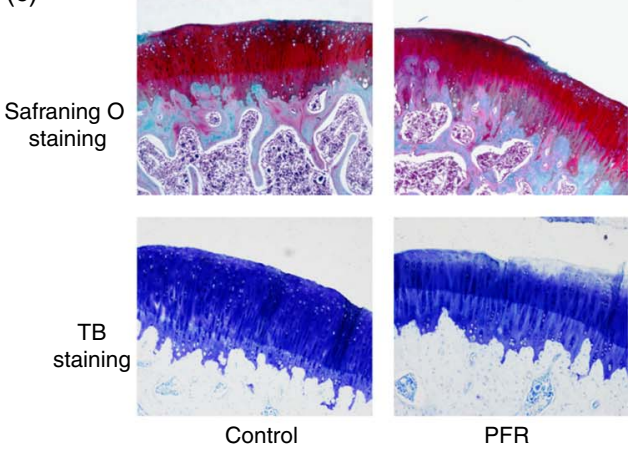

(d)

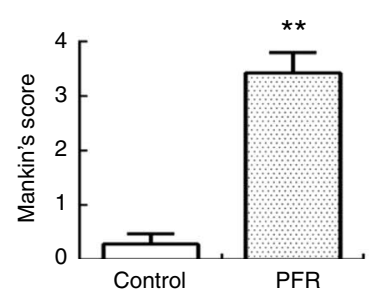

Fig. 2. Effects of prenatal food restriction (PFR) and a post-weaning high-fat diet on the ultrastructure of distal femoral articular cartilage in fetuses and offspring ( $\times 100)$. (a) Female fetal femoral knee cartilage sections were stained with safranin $O$. (b) Optical density analysis for safranin $O$ staining. (c) Female adult femoral knee cartilage sections were stained with safranin $\mathrm{O}$ and toluidine blue (TB). (d) Modified Mankin score. Values are means ( $n$ 8), with standard errors represented by vertical bars. ${ }^{* *}$ Mean value was significantly different from that of the control group $(P<0.01)$. For a colour figure, see the online version of the paper. 
layers in the control group, whereas the cartilage of the PFR group stained lightly and unevenly (Fig. 2(c)). The modified Mankin's score of the PFR group was significantly lower ( $P<0 \cdot 01$, Fig. 2(d)). The components of the modified Mankin's score showed that alteration was concentrated in the matrix staining, indicating that cartilage degradation of the PFR group may be the loss of the cartilage matrix.

\section{Gene and protein expression levels of articular cartilage in fetuses and adult offspring}

IGF-1 signalling pathway-related and cholesterol efflux-related gene expressions of female fetal femoral knee cartilage were detected using multiplex gene expression analysis (Fig. 3(a)). The detection of mRNA expression in the control and PFR groups showed that the IGF-1 signalling pathway (except $I G F-1 R$ ) and cartilage functional proteins Col2a1 and aggrecan had a declining trend, although there was no statistical difference. Regarding cholesterol-related mRNA expression, $L D L$ receptor was lower and PPARy had no change; the key gene of cholesterol efflux pathways, $L X R$, was lower significantly, although the other gene $A B C A 1$ was higher than that of the control group. Compared with the control group, the IGF-1 and Col2a1 protein expression levels of fetal articular cartilage in the PFR group were significantly decreased in the immunohistochemical assay $(P<0.05)$. The changes of protein expression were coincident with mRNA expression.

In adult rat offspring, the IGF-1 protein level in PFR females' articular cartilage was significantly lower than that of the control group $(P<0.05)$; the Col2a1 protein level in the PFR group was also low $(P<0.05)$. The LXR protein expression of articular cartilage in the PFR group was significantly lower than that of the control group $(P<0 \cdot 01)$. The protein level of ABCA1 in female offspring's cartilage showed no statistical difference between the two groups (Figs 3 and 4).

\section{Serum corticosterone, insulin-like growth factor-1 and cholesterol levels in fetuses and adult offspring}

In fetal rats, PFR increased fetal serum CORT levels $(P<0 \cdot 01)$ and decreased fetal serum IGF-1 levels significantly $(P<0 \cdot 05$, Fig. 5(a)). There was no statistical difference in fetal serum levels of $\mathrm{TCH}$, LDL-cholesterol and HDL-cholesterol between the two groups.

Serum CORT levels in PFR female offspring was significantly higher than that of the control group $(P<0 \cdot 05$, Fig. 5(b)). HFD increased serum IGF-1 levels in PFR female offspring $(P<0.05$, Fig. 5(b)). Serum TCH and LDL-cholesterol concentrations were decreased in PFR female offspring fed HFD $(P<0 \cdot 01$, Fig. 5(b)), and there was no significant difference in serum HDLcholesterol concentration between the two groups.

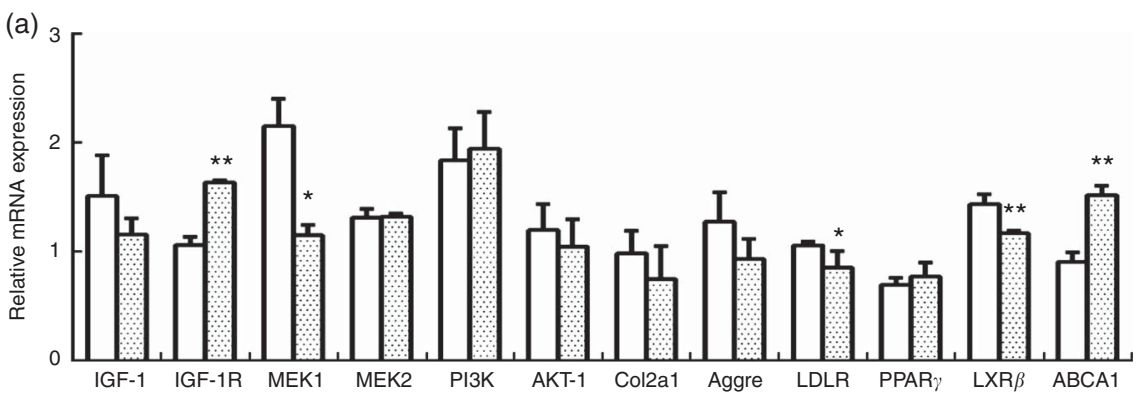

(b)
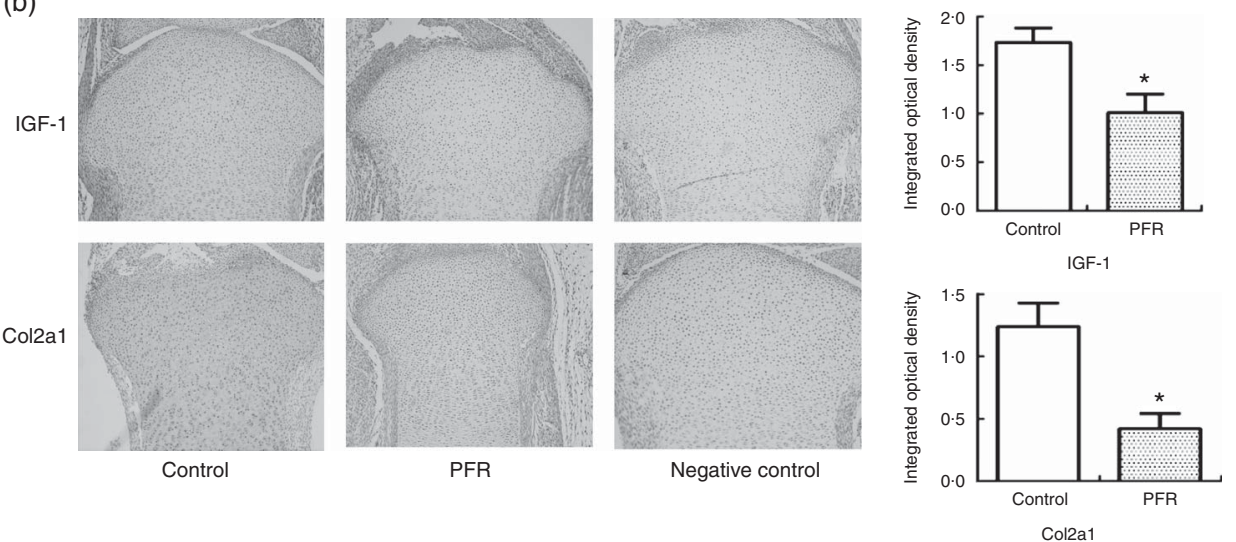

Fig. 3. Effects of prenatal food restriction (PFR; 䍘) on gene and protein expression in fetal articular cartilage. (a) mRNA expression level of insulin-like growth factor-1 (IGF-1), insulin-like growth factor-1 receptor (IGF-1R), mitogen-activated protein kinase 1 (MEK1), mitogen-activated protein kinase 2 (MEK2), phosphatidylinositol 3-kinase (PI3K), protein kinase B (Akt), collagen type lla1 (Col2a1), LDL receptor (LDLR), liver $\times$ receptor $\beta$ (LXR $\beta$ ), ATP-binding cassette transporter A1 (ABCA1) and aggrecan (Aggre), analysed by the GenomeLab GeXP Genetic System, normalised to glyceraldehyde 3-phosphate dehydrogenase (GAPDH), $\beta$-actin and tubulin. $\square$, Control group. (b) Immunohistochemical analysis of IGF-1 and Col2a1 expression in female fetal cartilage ( $\times 100)$. Values are means ( $n$ 8), with standard errors represented by vertical bars. Mean value was significantly different from that of the control group: ${ }^{\star} P<0.05,{ }^{\star \star} P<0.01$. 

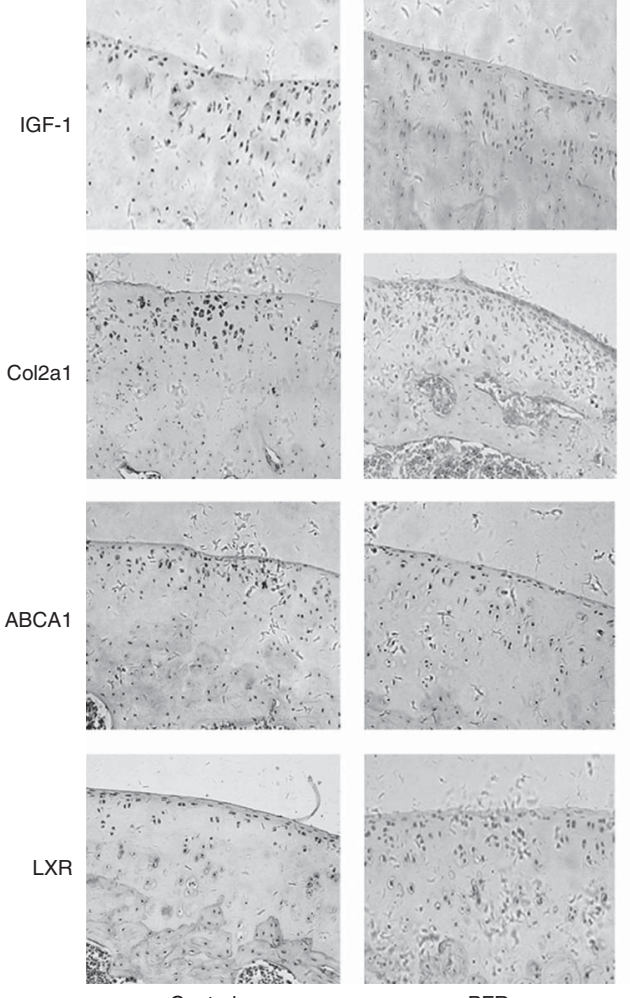

PFR
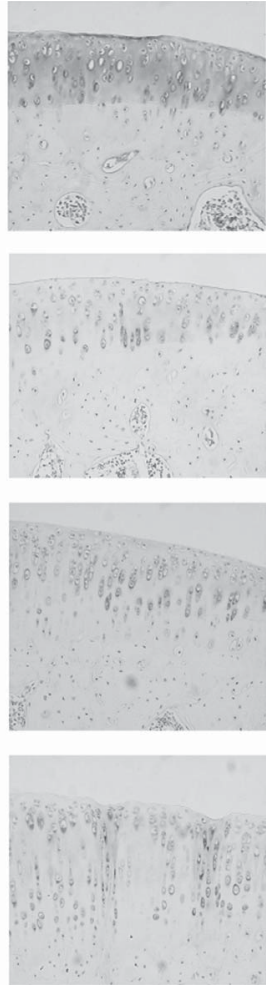

Negative control
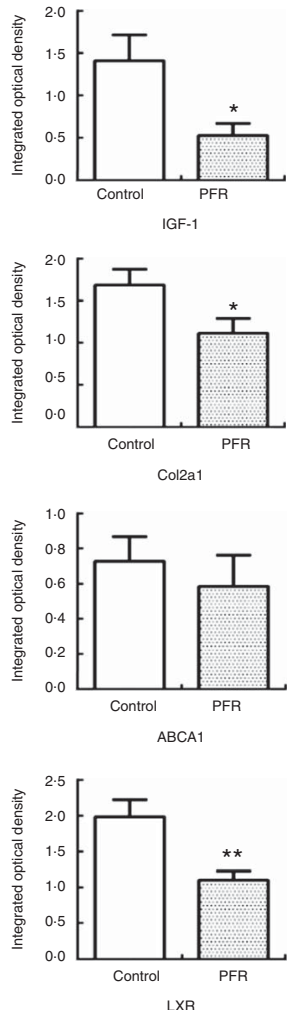

LXR

Fig. 4. Effects of prenatal food restriction (PFR) and a post-weaning high-fat diet on protein expression in adult offspring articular cartilage. Protein expression levels of insulin-like growth factor-1 (IGF-1) $\times 200$, collagen type lla1 (Col2a1) $\times 200$, liver $\times$ receptor $\beta$ (LXR) $\times 200$ and ATP-binding cassette transporter A1 (ABCA1) $\times 200$; the intensity of staining was determined by measuring the integral optical density in ten different fields for each sample. Values are means ( $n$ ), with standard errors represented by vertical bars. Mean value was significantly different from that of the control group: ${ }^{*} P<0.05,{ }^{\star *} P<0.01$.

\section{Discussion}

\section{Prenatal food-restriction-induced intra-uterine growth} retardation offspring cartilage dysplasia led to low quality in adults

The articular cartilage consists of chondrocytes and an extracellular matrix $(\mathrm{ECM})^{(43)}$. The ECM is mainly composed of Col2a1 and aggrecan. Col2a1 forms a dense, cross-linked network and primarily contributes to the tensile and shear properties of the tissue. Aggrecan consists of numerous carboxyl and sulphate groups that become negatively charged when dissolved in interstitial fluid. The presence of these charges gives rise to repulsive forces and osmotic gradients so that a swelling pressure exists within the tissue. The loss of Col2a1 and aggrecan is also a major hallmark and may significantly promote the occurrence and development of the pathological changes of $\mathrm{OA}^{(44)}$. In the present study, we found that PFR may result in reduction of safranin $O$ staining in offspring with IUGR. Safranin $O$ staining and TB staining were uneven and significantly reduced in adult rat offspring fed HFD; the Mankin's score further confirms these changes. These results show that PFR could result in a reduction of aggrecan both in fetuses and in adults. Meanwhile, the Col2a1 immunohistochemical staining was significantly lower in the PFR group compared with controls both in fetuses and in adults. Although the Mankin's score analyses showed that the pathological changes of articular cartilage in the PFR group did not meet OA criteria - the quality of articular cartilage was obviously poorer than that of the controls. Therefore, we can conclude that PFR could result in a reduction of Col2a1 and aggrecan, and that these influences were intra-uterine programming alterations and may be lifelong. The poor cartilage quality of the PFR rat tends to get worse under post-weaning HFD, enhancing the susceptibility to OA.

The poor quality of cartilage in prenatal food-restriction adult offspring fed an high-fat diet was associated with low-functionally programmed insulin-like growth factor-1 pathway and cholesterol efflux

In the present study, we found that PFR significantly decreased serum IGF-1 levels; meanwhile both mRNA and protein expressions of $I G F-1$ in the PFR fetal cartilage were significantly reduced, and its downstream gene MEK1 was decreased remarkably, which may inhibit chondrocyte proliferation. The expression levels of $P I 3 K$ and $A K T-1$ in the PFR-induced offspring with IUGR were still lower in articular cartilage. The mRNA expression levels of Col2a1 and aggrecan, which are the target genes of the IGF-1 signalling pathway, were significantly reduced. Immunohistochemistry analysis also confirmed that the protein expression of Col2a1 in the PFR group was significantly reduced when compared with the control group.

Under conditions of postnatal over-nutrition, offspring with IUGR usually exhibited rapid catch-up growth, directly shown 
(a)
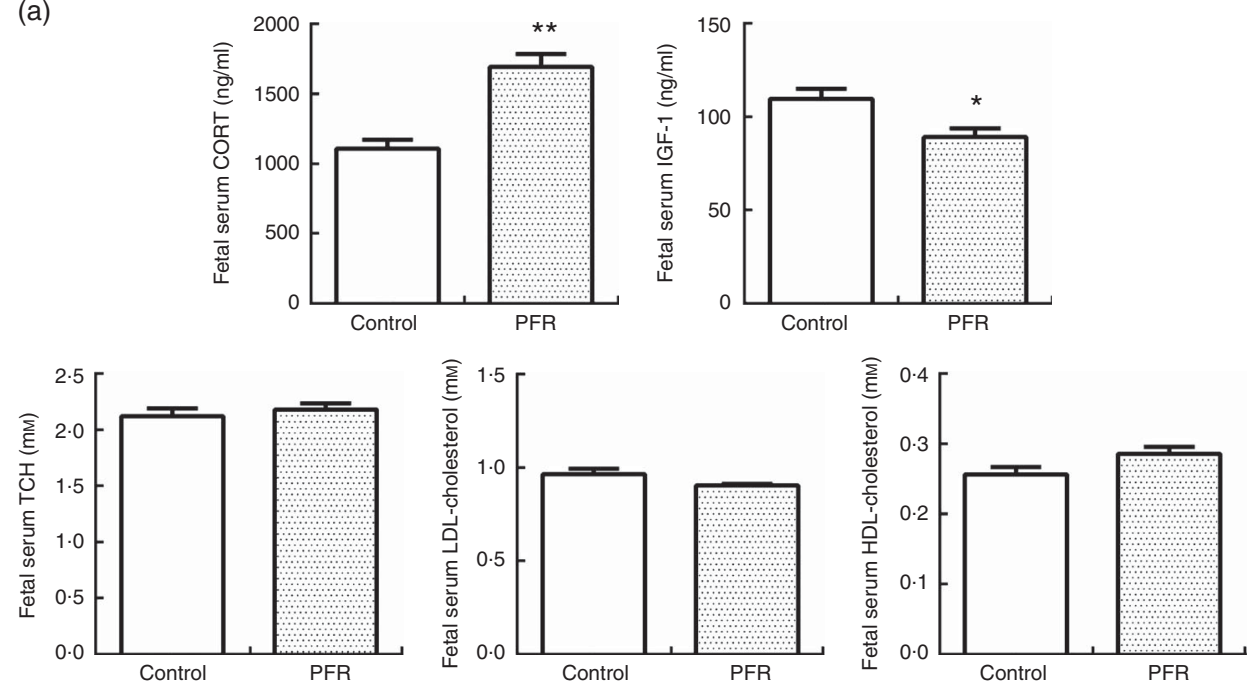

(b)
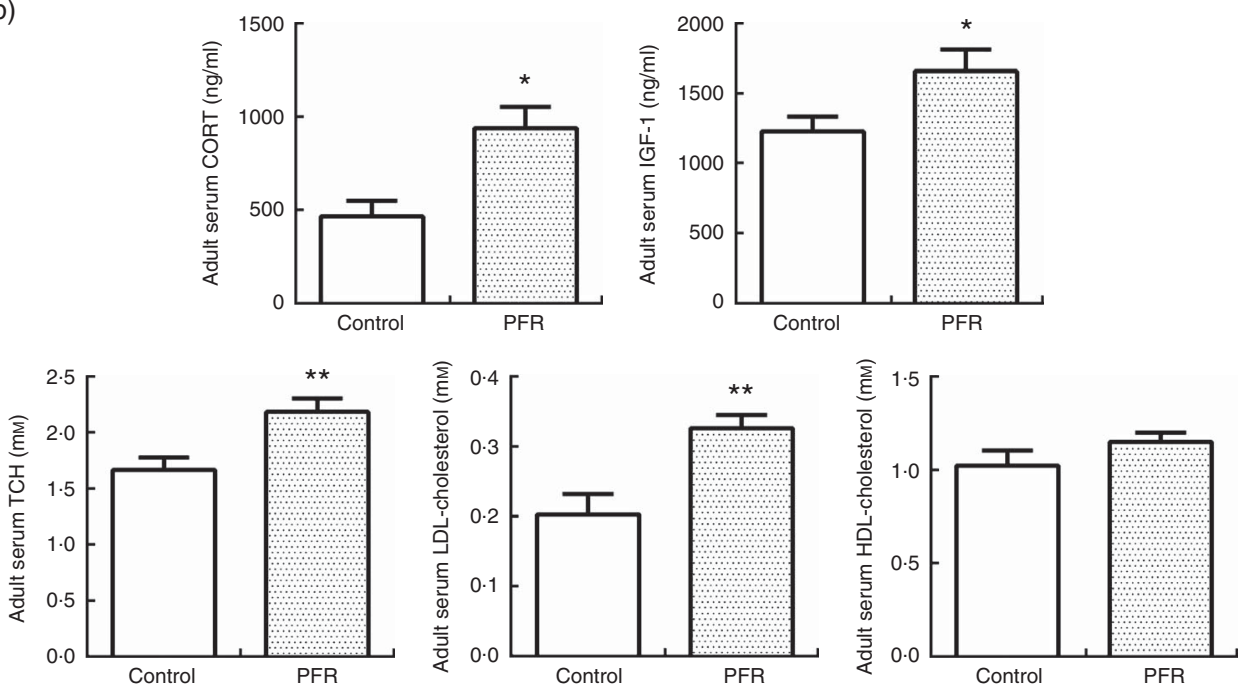

Fig. 5. Effects of prenatal food restriction (PFR) and a post-weaning high-fat diet on serum corticosterone (CORT), insulin-like growth factor-1 (IGF-1) and cholesterol levels in fetuses and adult offspring. (a) Female fetal serum concentration of CORT, IGF-1, total cholesterol (TCH), LDL-cholesterol and HDL-cholesterol. (b) Female adult offspring serum concentration of CORT, IGF-1, TCH, LDL-cholesterol and HDL-cholesterol. Values are means ( $n$ 8), with standard errors represented by vertical bars. Mean value was significantly different from that of the control group: ${ }^{\star} P<0.05,{ }^{\star *} P<0.01$.

as children's rapid growth and development, which increased susceptibility to adult MS, including obesity, insulin resistance, dyslipidaemia and so on ${ }^{(33,45)}$. Increased IGF-1 from the liver plays an important role in postnatal catch-up growth ${ }^{(46)}$. We reached similar conclusions that the growth rate in PFR adult offspring showed a significant increase and a significantly increased serum IGF-1 level. However, the articular cartilage is less affected by serum IGF-1, because adult articular cartilage is a vascular tissue, and the function of chondrocyte autocrine IGF-1 is still at a low functional level. These changes ultimately resulted in a persistent low expression of Col2a1 and further led to inadequate ECM and poor-quality cartilage.

The pathological basis of dyslipidaemia leading to OA is the accumulation of lipids in chondrocytes. Tsezou et $a l^{(47)}$ reported that lipid specifically accumulates in the superficial area of OA cartilage, and reduced expressions of $\operatorname{LXR} \alpha / \beta$ and ApoA1 have been found in human OA cartilage. Collins-Racie et $a l^{(48)}$ reached a similar result, that the expression of LXR was significantly lower in OA cartilage. In addition, Tsezou found that treatment of osteoarthritic chondrocytes with the LXR agonist TO-901317 significantly increased ApoA1 and ABCA1 expression levels, and cholesterol efflux decreased lipid deposits within osteoarthritic chondrocytes ${ }^{(48)}$. Therefore, LXR is an important mediator of cholesterol efflux in chondrocytes. In the present study, the results showed that LXR mRNA expression was decreased in PFR females' fetal articular cartilage, and that protein expression was still decreased in adult female PFR offspring. These results showed that PFR can affect the expression of LXR and further lead to the inhibition of cholesterol efflux and cartilage lesions. ABCA1 is regulated by the LXR gene; ABCA1 expression in the fetal cartilage was contrarily elevated because the fetal cholesterol metabolism may still have been in the normal or compensatory period. In adult female cartilage, the expression of ABCA1 was not changed because cartilage lesions 
were still well below OA levels. Meanwhile, the postnatal PFR female offspring fed HFD showed elevated serum TAG and LDL-cholesterol concentrations, which may enhance the accumulation of cholesterol in cartilage, further reducing cartilage quality and increasing the susceptibility to OA.

\section{Overexposure to maternal glucocorticoids results in the inhibition of the insulin-like growth factor-1 signalling pathway and cholesterol efflux in the offspring's cartilage}

GC is a vital metabolic hormone regulating intra-uterine fetal growth and development ${ }^{(49)}$. The GC in the fetus mainly comes from the mother rats, through the placenta to the fetus; physiologically, the placental barrier builds and maintains a GC concentration gradient (approximately 10-fold) between mother and fetus during pregnancy. Intra-uterine malnutrition may lead to fetal overexposure to maternal GC and induce dysfunction of the HPA axis by affecting the placental barrier ${ }^{(50)}$. In our previous study, we demonstrated that the effect of maternal food restriction on fetuses may be lifelong, which manifests as lower basal activity but enhanced sensitivity of the HPA axis to chronic stress, altering glucose and lipid metabolism ${ }^{(19)}$. It has been reported that GC could inhibit the IGF-1 signalling pathway expression in fetal epiphyseal chondrocytes ${ }^{(51)}$. In the present study, the PFR offspring exhibited an increased serum GC, and the elevated fetal serum GC may inhibit the function of the liver's IGF-1 secretion and decrease IGF-1 levels in the serum; meanwhile, the elevated fetal serum GC could also inhibit the autocrine function of IGF-1 in cartilage $^{(52)}$. PFR may lead to fetal overexposure to maternal GC, and the elevated serum GC may inhibit both the liver's and the cartilage's IGF-1 secretion, and this makes the endocrine and autocrine IGF-1 insufficient, ultimately leading to a reduced expression of the IGF-1 signalling pathway in the cartilage of PFR fetuses. Interestingly, the expression of IGF-1 was still decreased in the PFR adult offspring fed HFD. The consistent alteration in utero and adulthood revealed that articular cartilage IGF-1 pathway of IUGR fetuses by PFR may be low-functionally programmed caused by overexposure to maternal GC, which may finally result in inadequate EMC and poor-quality cartilage. This has revealed the mechanism of poor cartilage quality in PFR rats.

The role of maternal nutrition, especially maternal undernutrition, in the sensitivity of adults to disease remains unclear, but it is clear that GC plays a key role in this process ${ }^{(18,53,54)}$ Accumulating evidence has demonstrated that intra-uterine malnutrition may have long-term consequences on endocrine and metabolic function, which is mainly displayed by elevated serum cholesterol and LDL-cholesterol concentrations; male offspring showed an obvious increase in serum cholesterol levels ${ }^{(16,55,56)}$. Our results are consistent with the above conclusions - the abnormal expression of LXR in the cartilage of PFR offspring is relative to the GC/GR modulation ${ }^{(57)}$, but the exact mechanism calls for further study.

\section{Conclusions}

The present study demonstrated that PFR induces low quality of articular cartilage in adult female offspring fed HFD. Its underlying mechanisms may be associated with PFR-induced fetal overexposure to maternal GC and may further inhibit the cartilage's IGF-1 signalling pathway, ultimately leading to articular cartilage dysplasia; the intra-uterine low functional programming of the IGF-1 signalling pathway can persist to adulthood and eventually lead to a poor quality of articular cartilage in offspring, thus increasing their susceptibility to OA. In addition, the PFR could induce the suppression of the cholesterol efflux pathway by low-functionally programmed LXR and lead to dyslipidaemia and increased blood cholesterol. This is also one of the mechanisms of PFR, inducing low quality of articular cartilage in adult female offspring.

\section{Acknowledgements}

This study was supported by grants from the National Natural Science Foundation of China (no. 81220108026, 81430089, 81403027, 81371940) and the Special Research Fund for the Doctoral Program of Higher Education (no. 20130141110037).

Y. T. designed the entire research, analysed the data and wrote the paper. Y. W. carried out the animal experiments. Q. N. conducted the immunohistochemical experiments. Y. D. and J. L. carried out the molecular experiments; L. W. and L. S. carried out the analysis of blood samples; Y. L. was responsible for animal killing and sample collection. J. M. contributed to the study design, H. W. provided theoretical and experimental guidance, and L. C. designed the research, formulated reviewer's questions and supervised the entire study.

All the authors have no conflicts of interest.

\section{References}

1. Baker-LePain JC \& Lane NE (2010) Relationship between joint shape and the development of osteoarthritis. Curr Opin Rheumatol 22, 538-543.

2. Pitsillides AA \& Beier F (2011) Cartilage biology in osteoarthritis - lessons from developmental biology. Nat Rev Rheumatol 7, 654-663.

3. Jordan KM, Syddall H, Dennison EM, et al. (2005) Birthweight, vitamin $\mathrm{D}$ receptor gene polymorphism, and risk of lumbar spine osteoarthritis. J Rheumatol 32, 678-683.

4. Plotnikoff R, Karunamuni N, Lytvyak E, et al. (2015) Osteoarthritis prevalence and modifiable factors: a population study. BMC Public Health 15, 1195.

5. Aigner T \& Richter W (2012) OA in 2011: age-related $\mathrm{OA}$ - a concept emerging from infancy? Nat Rev Rheumatol 8, 70-72.

6. Szostak-Wegierek D \& Szamotulska K (2011) Fetal development and risk of cardiovascular diseases and diabetes type 2 in adult life. Med Wieku Rozwoj 15, 203-215.

7. Sohi G, Revesz A \& Hardy DB (2011) Permanent implications of intrauterine growth restriction on cholesterol homeostasis. Semin Reprod Med 29, 246-256.

8. Valsamakis G, Kanaka-Gantenbein C, Malamitsi-Puchner A, et al. (2006) Causes of intrauterine growth restriction and the postnatal development of the metabolic syndrome. Ann $N Y$ Acad Sci 1092, 138-147.

9. Gkretsi V, Simopoulou T \& Tsezou A (2011) Lipid metabolism and osteoarthritis: lessons from atherosclerosis. Prog Lipid Res 50, 133-140

10. Erb N, Pace AV, Douglas KM, et al. (2004) Risk assessment for coronary heart disease in rheumatoid arthritis and osteoarthritis. Scand J Rheumatol 33, 293-299. 
11. Calvet J, Orellana C, Larrosa M, et al. (2015) High prevalence of cardiovascular co-morbidities in patients with symptomatic knee or hand osteoarthritis. Scand J Rheumatol (epublication ahead of print version 27 August 2015).

12. Al-Arfaj AS (2003) Radiographic osteoarthritis and serum cholesterol. Saudi Med J 24, 745-747.

13. Singh G, Miller JD, Lee FH, et al. (2002) Prevalence of cardiovascular disease risk factors among US adults with selfreported osteoarthritis: data from the Third National Health and Nutrition Examination Survey. Am J Manag Care 8, S383-S391.

14. Philbin EF, Ries MD, Groff GD, et al. (1996) Osteoarthritis as a determinant of an adverse coronary heart disease risk profile. J Cardiovasc Risk 3, 529-533.

15. Lingas RI \& Matthews SG (2001) A short period of maternal nutrient restriction in late gestation modifies pituitary-adrenal function in adult guinea pig offspring. Neuroendocrinology 73, 302-311.

16. Desai M, Gayle D, Babu J, et al. (2007) The timing of nutrient restriction during rat pregnancy/lactation alters metabolic syndrome phenotype. Am J Obstet Gynecol 196, 551-555.

17. Erhuma A, Salter AM, Sculley DV, et al. (2007) Prenatal exposure to a low-protein diet programs disordered regulation of lipid metabolism in the aging rat. Am J Physiol Endocrinol Metab 292, E1702-E1714.

18. Langley-Evans SC, Phillips GJ, Benediktsson R, et al. (1996) Protein intake in pregnancy, placental glucocorticoid metabolism and the programming of hypertension in the rat. Placenta 17, 169-172.

19. Reynolds RM (2010) Corticosteroid-mediated programming and the pathogenesis of obesity and diabetes. I Steroid Biochem Mol Biol 122, 3-9.

20. Liang G, Chen M, Pan XL, et al. (2011) Ethanol-induced inhibition of fetal hypothalamic-pituitary-adrenal axis due to prenatal overexposure to maternal glucocorticoid in mice. Exp Toxicol Pathol 63, 607-611.

21. Chen M, Wang T, Liao ZX, et al. (2007) Nicotine-induced prenatal overexposure to maternal glucocorticoid and intrauterine growth retardation in rat. Exp Toxicol Pathol 59, 245-251.

22. Xu D, Chen M, Pan XL, et al. (2011) Dexamethasone induces fetal developmental toxicity through affecting the placental glucocorticoid barrier and depressing fetal adrenal function. Environ Toxicol Pharmacol 32, 356-363.

23. Xu D, Zhang B, Liang G, et al. (2012) Caffeine-induced activated glucocorticoid metabolism in the hippocampus causes hypothalamic-pituitary-adrenal axis inhibition in fetal rats. PLOS ONE 7, e44497.

24. Zhang L, Xu D, Zhang B, et al. (2013) Prenatal food restriction induces a hypothalamic-pituitary-adrenocortical axisassociated neuroendocrine metabolic programmed alteration in adult offspring rats. Arch Med Res 44, 335-345.

25. Giustina A, Mazziotti G \& Canalis E (2008) Growth hormone, insulin-like growth factors, and the skeleton. Endocr Rev 29, 535-559.

26. Starkman BG, Cravero JD, Delcarlo M, et al. (2005) IGF-I stimulation of proteoglycan synthesis by chondrocytes requires activation of the PI 3-kinase pathway but not ERK MAPK. Biochem J 389, 723-729.

27. Wang Y, Nishida S, Sakata T, et al. (2006) Insulin-like growth factor-I is essential for embryonic bone development. Endocrinology 147, 4753-4761.

28. Perrini S, Laviola L, Carreira MC, et al. (2010) The GH/IGF1 axis and signaling pathways in the muscle and bone: mechanisms underlying age-related skeletal muscle wasting and osteoporosis. J Endocrinol 205, 201-210.
29. Laviola L, Natalicchio A, Perrini S, et al. (2008) Abnormalities of IGF-I signaling in the pathogenesis of diseases of the bone, brain, and fetoplacental unit in humans. Am J Physiol Endocrinol Metab 295, E991-E999.

30. Hochberg Z (2002) Mechanisms of steroid impairment of growth. Horm Res 58, Suppl. 1, 33-38.

31. Delany AM, Durant D \& Canalis E (2001) Glucocorticoid suppression of IGF I transcription in osteoblasts. Mol Endocrinol 15, 1781-1789.

32. Zanotti I, Favari E \& Bernini F (2012) Cellular cholesterol efflux pathways: impact on intracellular lipid trafficking and methodological considerations. Curr Pharm Biotechnol 13, 292-302.

33. Morrison JL, Duffield JA, Muhlhausler BS, et al. (2010) Fetal growth restriction, catch-up growth and the early origins of insulin resistance and visceral obesity. Pediatr Nephrol 25 , 669-677.

34. Dulloo AG (2008) Thrifty energy metabolism in catch-up growth trajectories to insulin and leptin resistance. Best Pract Res Clin Endocrinol Metab 22, 155-171.

35. Price MD \& Herndon JH (2009) Gender differences in osteoarthritis. Menopause 16, 624-625.

36. Boyan BD, Tosi L, Coutts R, et al. (2012) Sex differences in osteoarthritis of the knee. J Am Acad Orthop Surg 20, 668-669.

37. Wang L, Shen L, Ping J, et al. (2014) Intrauterine metabolic programming alteration increased susceptibility to nonalcoholic adult fatty liver disease in prenatal caffeineexposed rat offspring. Toxicol Lett 224, 311-318.

38. Carbone DL, Zuloaga DG, Hiroi R, et al. (2012) Prenatal dexamethasone exposure potentiates diet-induced hepatosteatosis and decreases plasma IGF-I in a sex-specific fashion. Endocrinology 153, 295-306.

39. Xia LP, Shen L, Kou H, et al. (2014) Prenatal ethanol exposure enhances the susceptibility to metabolic syndrome in offspring rats by HPA axis-associated neuroendocrine metabolic programming. Toxicol Lett 226, 98-105.

40. Mankin HJ (1971) Biochemical and metabolic aspects of osteoarthritis. Orthop Clin North Am 2, 19-31.

41. Henson FM \& Vincent TA (2008) Alterations in the vimentin cytoskeleton in response to single impact load in an in vitro model of cartilage damage in the rat. BMC Musculoskelet Disord 9, 94.

42. Shen L, Liu Z, Gong J, et al. (2014) Prenatal ethanol exposure programs an increased susceptibility of non-alcoholic fatty liver disease in female adult offspring rats. Toxicol Appl Pharmacol 274, 263-273.

43. Cohen NP, Foster RJ \& Mow VC (1998) Composition and dynamics of articular cartilage: structure, function, and maintaining healthy state. J Orthop Sports Phys Ther 28, 203-215.

44. Johnston SA (1997) Osteoarthritis. Joint anatomy, physiology, and pathobiology. Vet Clin North Am Small Anim Pract 27, 699-723.

45. Proos L \& Gustafsson J (2012) Is early puberty triggered by catch-up growth following undernutrition? Int J Environ Res Public Health 9, 1791-1809.

46. Tosh DN, Fu Q, Callaway CW, et al. (2010) Epigenetics of programmed obesity: alteration in IUGR rat hepatic IGF1 mRNA expression and histone structure in rapid vs. delayed postnatal catch-up growth. Am J Physiol Gastrointest Liver Physiol 299, G1023-G1029.

47. Tsezou A, Iliopoulos D, Malizos KN, et al. (2010) Impaired expression of genes regulating cholesterol efflux in human osteoarthritic chondrocytes. J Orthop Res 28, 1033-1039.

48. Collins-Racie LA, Yang Z, Arai M, et al. (2009) Global analysis of nuclear receptor expression and dysregulation in human osteoarthritic articular cartilage: reduced LXR signaling 
contributes to catabolic metabolism typical of osteoarthritis. Osteoarthritis Cartilage 17, 832-842.

49. McNeil CJ, Nwagwu MO, Finch AM, et al. (2007) Glucocorticoid exposure and tissue gene expression of 11beta HSD-1, 11beta HSD-2, and glucocorticoid receptor in a porcine model of differential fetal growth. Reproduction 133, 653-661.

50. Metges CC, Lang IS, Hennig U, et al. (2012) Intrauterine growth retarded progeny of pregnant sows fed high protein: low carbohydrate diet is related to metabolic energy deficit. PLOS ONE 7, e31390.

51. Fernandez-Cancio M, Esteban C, Carrascosa A, et al. (2008) IGF-I and not IGF-II expression is regulated by glucocorticoids in human fetal epiphyseal chondrocytes. Growth Horm IGF Res 18, 497-505.

52. Olney RC (2009) Mechanisms of impaired growth: effect of steroids on bone and cartilage. Horm Res 72, Suppl. 1, 30-35.

53. Whorwood CB, Firth KM, Budge H, et al. (2001) Maternal undernutrition during early to midgestation programs tissuespecific alterations in the expression of the glucocorticoid receptor, 11beta-hydroxysteroid dehydrogenase isoforms, and type 1 angiotensin ii receptor in neonatal sheep. Endocrinology 142, 2854-2864.

54. Bertram C, Trowern AR, Copin N, et al. (2001) The maternal diet during pregnancy programs altered expression of the glucocorticoid receptor and type 2 11beta-hydroxysteroid dehydrogenase: potential molecular mechanisms underlying the programming of hypertension in utero. Endocrinology 142, 2841-2853.

55. Szitanyi P, Hanzlova J \& Poledne R (2000) Influence of intrauterine undernutrition on the development of hypercholesterolemia in an animal model. Physiol Res 49, 721-724.

56. Choi GY, Tosh DN, Garg A, et al. (2007) Gender-specific programmed hepatic lipid dysregulation in intrauterine growth-restricted offspring. Am J Obstet Gynecol 196, 471-477.

57. Steffensen KR, Holter E, Alikhani N, et al. (2003) Glucocorticoid response and promoter occupancy of the mouse LXRalpha gene. Biochem Biophys Res Commun 312, 716-724. 